Marco Tackenberg, Presse- und Informationsdienst der Ärztegesellschaft des Kantons Bern
Im heissen Sommer 2003 traf sich der Vorstand der Ärztegesellschaft des Kantons Bern auf dem Gurten, dem Hausberg der Bundesstadt, zur jährlichen Klausurtagung. Traktandiert war ein einziges Thema, aber das hatte es in sich: Wie würden Ärztinnen und Ärzte das Gesundheitswesen reformieren? Konnte es gelingen, eine so heterogene Gruppe wie den Vorstand einer grossen Kantonalgesellschaft auf gemeinsame Positionen zu verpflichten? Gerade unter den Leistungserbringern ist der Glaube verbreitet, die eigene Gilde ringe sich kaum zu gemeinsamen Positionen durch: Spezialisten hätten andere Interessen als Grundversorger, Ärzte auf dem Land andere Sorgen als jene in Städten und Agglomerationen. Die jungen Ärzte seien vom Zulassungsstopp betroffen, während die älteren mehr an TARMED und Kontrahierungszwang interessiert seien. Und nicht zuletzt verträten Ärztinnen ihre ganz eigenen Anliegen, welche von den männlichen Berufskollegen kaum verstanden, geschweige denn geteilt würden.

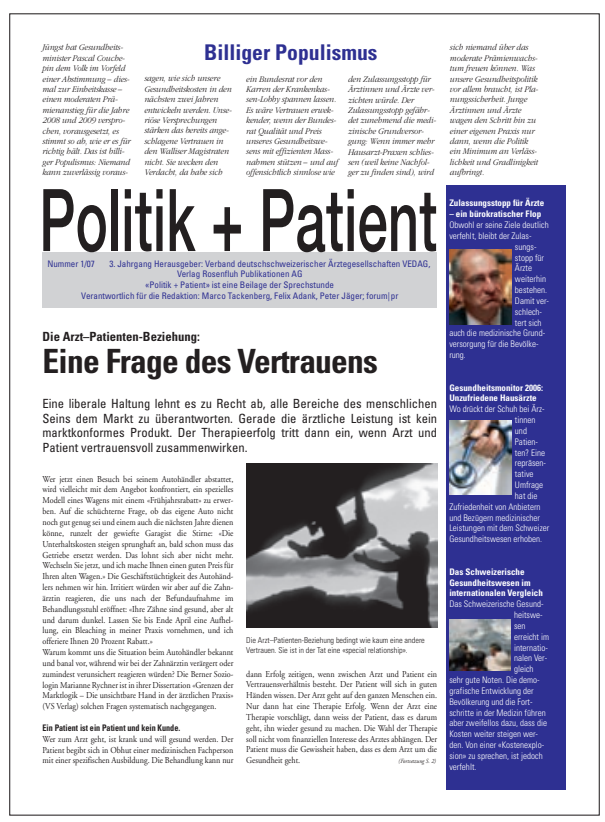

\section{Gesundheitspolitische Leitsätze}

Die Vorbereitungsarbeiten zur Klausur brachten Erstaunliches zutage. Etwa zur selben Zeit wie die Ärztegesellschaft des Kantons Bern (BEKAG) hatten der Verband deutschschweizerischer Ärztegesellschaften (VEDAG) und die Société médicale de la Suisse romande (SMSR) eigene Thesenpapiere und Leitsätze erarbeitet. Ein Vergleich der beiden Papiere mit den aus der Klausur hervorgegangenen «Gesundheitspolitischen Positionen» der Berner Ärzte zeigte, dass die Übereinstimmungen weit grösser waren als die Differenzen. Ein paar Punkte seien herausgegriffen:

Alle drei Ärzteorganisationen waren sich einig, dass im Zentrum der beruflichen Tätigkeit das Wohl des Patienten und das Vertrauensverhältnis zwischen Arzt und Patient stehen müssen. Patienten sollen die Hilfe erhalten, die medizinisch notwendig ist. Die Positionspapiere sprechen sich dabei gegen die Rationierung medizinischer Leistungen aus. Auch sollen Patienten unabhängig von ihrem sozialen Status den Arzt frei wählen können.

Insbesondere VEDAG und BEKAG betonen aber auch, dass der Leistungskatalog der obligatorischen Krankenversicherung auf das medizinisch Notwendige beschränkt werden soll. Die gesetzlichen Anforderungen nach Wirksamkeit, Zweckmässigkeit und Wirtschaftlichkeit müssen eingehalten werden. Alle drei Organisationen erklären jedoch auch, dass neue Heilmethoden und Innovationen in den Leistungskatalog aufgenommen werden sollen.

\section{Konsens grösser als vermutet}

Die Tatsache, dass die Übereinstimmungen grösser waren als die Differenzen, bewog den Präsidenten der Ärztegesellschaft des Kantons Bern, Jürg Schlup, das Projekt einer gesundheitspolitischen Zeitung anzupacken. Es war die Geburtsstunde von «Politik + Patient». Die Zeitung, an welcher sich heute 15 kantonale Ärztegesellschaften beteiligen, bringt seit vier Jahren die Sicht der Ärztinnen und Ärzte in die öffentliche Debatte ein.

Für die Ärztegesellschaft des Kantons Bern ergeben sich ermutigende Lehren aus der Klausur vom Sommer 2003: Die politischen Gemeinsamkeiten unter den Ärztinnen und Ärzten sind grösser als vermutet. Sie erlauben das gemeinsame Verfolgen gesundheitspolitischer Anliegen. Zudem geniessen Ärztinnen und Ärzte eine hohe Glaubwürdigkeit in der Bevölkerung. Dies, zusammengenommen, ist ermutigend. Es erlaubt, den kommenden gesundheitspolitischen Herausforderungen gemeinsam mit konkreten Projekten zu begegnen. 